Pacific Journal of Mathematic 


\title{
NONLINEAR DIFFERENTIAL EQUATIONS WITH MONOTONE SOLUTIONS
}

\author{
KURT KREITH
}

\begin{abstract}
The differential equation $d y^{4} / d t^{4}-y=0$ has as a fundamental set of solutions $\sin t, \cos t, e^{t}$, and $e^{-t}$. The latter of these is distinguished by the properties of being positive and strictly decreasing to zero as $t \rightarrow \infty$. As such, $e^{-t}$ is the prototype of the "monotone solution" whose existence will be demonstrated for a large class of nonlinear differential equations of even order.
\end{abstract}

Our method will be restricted to differential equations of order $2 n$ which can be written as second order systems of the form

$$
\boldsymbol{x}^{\prime \prime}=\boldsymbol{f}(\boldsymbol{x}, t)
$$

where $\boldsymbol{x} \in \boldsymbol{R}^{n}$ and $\boldsymbol{f}$ is a continuous function from $\boldsymbol{R}^{n} \times[0, \infty)$ into $\boldsymbol{R}^{n}$ satisfying other conditions to be formulated in $\S 2$. Without resolving the question of what scalar equations allow such a representation, it is clear that our considerations will include nonselfadjoint linear fourth order equations (see [5]), equations of the form

$$
\begin{aligned}
\left.\left(p_{1} y^{\prime \prime}\right)\right)^{\prime \prime} & =f_{2}\left(y, y^{\prime \prime}, t\right) \\
{\left[p_{2}\left(p_{1} y^{\prime \prime}\right)^{\prime \prime}\right]^{\prime \prime} } & =f_{3}\left(y, y^{\prime \prime}, y^{i v}, t\right),
\end{aligned}
$$

and similar equations of higher order.

In case (1.1) is linear and

$$
\boldsymbol{x}^{\prime \prime}=A(t) \boldsymbol{x}
$$

where $A(t)=\left(a_{i j}(t)\right)$ is a continuous $n \times n$ matrix, criteria for the existence of monotone solutions of (1.2) are well known. In particular, by letting $\boldsymbol{w}=-^{\prime} \boldsymbol{x},(1.2)$ can be written as a first order system of the form

$$
\left(\begin{array}{l}
\boldsymbol{x} \\
\boldsymbol{w}
\end{array}\right)^{\prime}=-\left(\begin{array}{cc}
0 & I \\
A(t) & 0
\end{array}\right)\left(\begin{array}{l}
\boldsymbol{x} \\
\boldsymbol{w}
\end{array}\right)
$$

According to Hartman [2; Ch 14, Theorem 2.1], the condition $a_{i j}(t) \geqq 0$ for $1 \leqq i, j \leqq n$, and $0 \leqq t<\infty$ assures the existence of a nontrivial solution of (1.3) for which $x_{i}(t) \geqq 0$ and $x_{i}^{\prime}(t) \leqq 0$ for $1 \leqq i \leqq n$ and $0 \leqq t<\infty$. Since $\boldsymbol{x}(t)$ also satisfies (1.2), these results readily carry over to linear second order systems.

Nonlinear problems of the form (1.1) have also been studied by Hartman and Wintner [3] in terms of the related first order systems. 
However their method is based on the assumption that solutions of (1.1) can be extended to the semi-infinite interval $[0, \infty)$, and this requires some restrictions on $\boldsymbol{f}(\boldsymbol{x}, t)$ as $|\boldsymbol{x}| \rightarrow \infty$. Our methods are essentially different and require no restrictions on the growth of $f$ for large values of $\boldsymbol{x}$.

For the sake of clarity, the basic ideas are developed in $\S 2$ for $n=2$. In $\S 3$ a theorem of Sperner [1] is used to generalize these results to the case $n \geqq 2$, while some applications to scalar equations are discussed in $\S 4$.

As a final introductory remark we note that the nonoscillatory equation $y^{\prime \prime}-y=0$, with fundamental solutions $e^{t}$ and $e^{-t}$, is frequently used to motivate the concept of "principal solution". Here $e^{-t}$ is singled out, not because it is monotone but because it is in an appropriate sense "smaller" than all other linearly independent solutions. The question therefore arises as to why $e^{-t}$ should not be characterized as a principal solution of $y^{(i v)}-y=0$ rather than a monotone solution and, more generally, why the distinguished solutions of higher order equations to be considered below should not be called "principal solutions".

Several answers can be given in the context of fourth order equations. First, $y^{(i v)}-y=0$ has both oscillatory and nonoscillatory solutions, while in the second order case principal solutions are defined only for nonoscillatory equations. Accordingly, we do not have " $\lim _{t \rightarrow \infty} e^{-t} / y(t)=0$ for any linearly independent solution $y(t)$," whereas such a condition characterizes principal solutions of second order equations. Furthermore, there is an elegant generalization of the scalar theory of principal solutions to disconjugate Hamiltonian systems (see for example [6]). However, since the equation $y^{(i v)}-y=0$ is not $2-2$ disconjugate in any interval $[a, \infty)$, the corresponding Hamiltonian system will also not be disconjugate in such intervals $[a, \infty)$ (see for example [5], $\mathrm{Ch} 6$ ). It follows that the systems theory of principal solutions does not apply to $y^{(i v)}-y=0$. For these reasons it seems appropriate to consider the equation $y^{(i v)}+4 y=0$ with solutions $e^{-t} \sin t$ and $e^{-t} \cos t$ as the motivating example for principal solutions of 2-2 disconjugate fourth order equations and to avoid this terminology in problems motivated by the equation $y^{(i v)}-y=0$.

2. Systems in $\boldsymbol{R}^{2}$. In considering second order differential systems of the form

$$
\boldsymbol{x}^{\prime \prime}=\boldsymbol{f}(\boldsymbol{x}, t)
$$

we shall require $f$ to be sufficiently regular in $R^{2} \times[0, \infty)$ to assure the existence, uniqueness, and stability of solutions of the initial 
value problem

$$
\boldsymbol{x}(a)=\boldsymbol{x}_{a} ; \quad \boldsymbol{x}^{\prime}(a)=\boldsymbol{v}_{a}
$$

for all $a \geqq 0$ and to assure that solutions of (2.1) can be continued until some component of $\boldsymbol{x}(t)$ becomes unbounded. In most instance we shall consider (2.2) with $a=0$. The interval of existence of a solution of (2.1) will be denoted by $[\alpha, \omega)$ where we allow $\omega=\infty$. Writing $\boldsymbol{v}>\mathbf{0}$ in case all components of a vector $v$ satisfy $v_{i}>0$ and writing $\boldsymbol{v}>\boldsymbol{w}$ in case $\boldsymbol{v}-\boldsymbol{w}>\boldsymbol{0}$, we formulate three additional conditions which will be assumed throughout:

(A) $\boldsymbol{f}(\boldsymbol{x}, t)>\mathbf{0}$ whenever $\boldsymbol{x}>\mathbf{0}$ and $\boldsymbol{f}(\boldsymbol{0}, t)=\mathbf{0}$;

(B) $f_{i}(\boldsymbol{x}, t)>0$ whenever $x_{i}=0$ and $x_{j}>0$ for all $j \neq i$;

(C) for every initial value $\left(\boldsymbol{x}_{0}, t_{0}\right)>0$ and every $T<\infty, \boldsymbol{f}(\boldsymbol{x}, t)$ is bounded in

$$
\left\{\boldsymbol{x} \mid \boldsymbol{0} \leqq \boldsymbol{x} \leqq \boldsymbol{x}_{0}\right\} \times\left\{t \mid t_{0} \leqq t \leqq T\right\}
$$

By way of example of a large class of systems satisfying these conditions in $\boldsymbol{R}^{2}$, we cite

$$
\begin{aligned}
& y^{\prime \prime}=a(t)|y|^{\alpha-1} y+b(t)|z|^{\beta-1} z \\
& z^{\prime \prime}=c(t)|y|^{\gamma-1} y+d(t)|z|^{j-1} z
\end{aligned}
$$

where $\alpha, \beta, \gamma$, and $\delta$ are nonnegative constants (or nonnegative continuous functions of $t$ ) and the coefficients are continuous functions satisfying

$$
a(t) \geqq 0, \quad b(t)>0, \quad c(t)>0, \quad d(t) \geqq 0
$$

in $[0, \infty)$.

It will be convenient to think of systems such as (2.1) and (2.3) as equations governing the motion of a particle of unit mass in a force field given by $\boldsymbol{f}$. Conditions (A) and (B) can then be interpreted as requiring that this force field be "repulsive" in the closed first $n$-tant, $\bar{I}=\{\boldsymbol{x} \mid \boldsymbol{x} \geqq 0\}$, except at $\boldsymbol{x}=\underline{0}$. The role of condition (C) will become apparent in Lemma 2.1 below.

In discussing the initial position and direction of such a particle, we define

$$
\left|\boldsymbol{x}_{0}\right|=\sum_{i=1}^{n}\left|x_{i}(0)\right| ; \quad \theta_{i}(0)=\frac{x_{i}(0)}{\left|\boldsymbol{x}_{0}\right|}
$$

and

$$
\left|\boldsymbol{v}_{0}\right|=\sum_{i=1}^{n}\left|x_{i}^{\prime}(0)\right| ; \quad \varphi_{i}(0)=\frac{x_{i}^{\prime}(0)}{\left|v_{0}\right|}
$$


Then initial position can be specified by $\boldsymbol{x}_{0}$ or by $\left|\boldsymbol{x}_{0}\right|$ and $\boldsymbol{\theta}_{0}$ while initial direction is specified by $\boldsymbol{v}_{0}$ or by $\left|\boldsymbol{v}_{0}\right|$ and $\boldsymbol{\phi}_{0}$. We note that $\left|\boldsymbol{\theta}_{0}\right|=\left|\boldsymbol{\Phi}_{0}\right|=\sum_{i=1}^{n} \theta_{i}(0)=\sum_{i=1}^{n} \varphi_{i}(0)=1$. Our first lemma asserts that for each $\boldsymbol{x}_{0}$ in the first $n$-tant and every initial direction $\boldsymbol{\phi}_{0}<0$ we can choose an initial speed $\left|\boldsymbol{v}_{0}\right|$ sufficiently large so that the particle must leave $\bar{I}$ in finite time. It will be useful to let $C\left(t ; \boldsymbol{x}_{0},\left|\boldsymbol{v}_{0}\right|, \boldsymbol{\phi}_{0}\right)$ denote the trajectory determined in $\boldsymbol{R}^{n}$ by the solution $\boldsymbol{x}(t)$ of (2.1) satisfying $\boldsymbol{x}(0)=\boldsymbol{x}_{0}, \boldsymbol{x}^{\prime}(0)=\left|\boldsymbol{v}_{0}\right| \boldsymbol{\phi}_{0}$.

Lemma 2.1. For every $\boldsymbol{x}_{0}>0$ and $\boldsymbol{\phi}_{0} \leqq 0$ there exists $s\left(\boldsymbol{x}_{0}, \boldsymbol{\phi}_{0}\right)$ so that $\left|\boldsymbol{v}_{0}\right| \geqq s$ implies that $C\left(t ; \boldsymbol{x}_{0},\left|\boldsymbol{v}_{0}\right|, \boldsymbol{\phi}_{0}\right)$ exits the closed first $n$-tant $\bar{I}$ in finite time.

Proof. For sufficiently small $t>0$ a solution $x(t)$ of (2.1) satisfies

$$
x_{i}(t)=x_{i}(0)+x_{i}^{\prime}(0) t+\int_{0}^{t} \int_{0}^{\tau} f_{i}(\boldsymbol{x}(\sigma), \sigma) d \sigma d \tau ; \quad i=1, \cdots, n,
$$

and this solution can be continued until some component becomes unbounded. Fixing a finite $T>0,(\mathrm{C})$ assures the existence of a constant $\boldsymbol{k}>0$ such that $\boldsymbol{f}(\boldsymbol{x}, t)<\boldsymbol{k}$ and

$$
x_{i}(t) \leqq x_{i}(0)+x_{i}^{\prime}(0) t+\frac{k_{i}}{2} t^{2} ; \quad i=1, \cdots, n
$$

as long as $0 \leqq x(t) \leqq x_{0}$ and $0 \leqq t \leqq T$. Recalling that $x_{i}^{\prime}(0)=$ $\left|\boldsymbol{v}_{0}\right| \varphi_{i}(0)<0$, we choose $s$ sufficiently large so that the $n$ binomials $x_{i}(0)+s \varphi_{i}(0) t+\left(k_{i} / 2\right) t^{2}$ all have zeros in $[0, T]$. Then it follows from (2.4) that $C\left(t ; \boldsymbol{x}_{0},\left|v_{0}\right| \boldsymbol{\phi}_{0}\right)$ exits $I$ in $[0, T]$ whenever $\left|v_{0}\right| \geqq s$.

Since $\boldsymbol{f}(\boldsymbol{x}, t)>0$ whenever $\boldsymbol{x}>0$, (2.4) implies that solutions of (2.1) satisfying $\boldsymbol{x}(0)>0$ and $\boldsymbol{x}^{\prime}(0)=0$ satisfy $\boldsymbol{x}(t)>0$ in $[0, \omega)$, their interval of existence. Thus it follows from Lemma 2.1 that to each $\boldsymbol{x}_{0}>\mathbf{0}$ and initial direction $\boldsymbol{\phi}_{0}<\mathbf{0}$ there corresponds a "critical speed"

$$
\boldsymbol{s}_{c}\left(\boldsymbol{x}_{0}, \boldsymbol{\phi}_{0}\right)=\inf \left\{\left|\boldsymbol{v}_{0}\right| \mid C\left(t ; \boldsymbol{x}_{0},\left|\boldsymbol{v}_{0}\right|, \boldsymbol{\Phi}_{0}\right) \text { exits } \bar{I} \text { in finite time }\right\} .
$$

Our objective is to show the existence of a "critical initial position" $\boldsymbol{x}_{c}$ and "critical initial direction" $\boldsymbol{\phi}_{c}$ for which $C\left(t ; \boldsymbol{x}_{c}, s_{c}\left(\boldsymbol{x}_{c}, \boldsymbol{\phi}_{c}\right), \boldsymbol{\phi}_{c}\right)$ is a monotone trajectory satisfying $\boldsymbol{x}(t)>\mathbf{0}$ and $\boldsymbol{x}^{\prime}(t)<0$ as $t \rightarrow \infty$. To that end we begin by restricting our attention to the case $n=2$ and examine the critical trajectories $C\left(t ; \boldsymbol{x}_{0}, \boldsymbol{s}_{c}\left(\boldsymbol{x}_{0}, \boldsymbol{\Phi}_{0}\right), \boldsymbol{\Phi}_{0}\right)$ in $\boldsymbol{R}^{2}$. The extension to $n>2$ will be considered in $\S 3$.

For $n=2$ we write $\boldsymbol{x}(t)=(y(t), z(t))$ and let $I$ denote the open first quadrant in the $(y, z)$-plane. The components of $C\left(t ; \boldsymbol{x}_{0},\left|\boldsymbol{v}_{0}\right|, \boldsymbol{\phi}_{0}\right)$ are now denoted by $y\left(t ; \boldsymbol{x}_{0},\left|\boldsymbol{v}_{0}\right|, \boldsymbol{\phi}_{0}\right)$ and $z\left(t ; \boldsymbol{x}_{0},\left|\boldsymbol{v}_{0}\right|, \boldsymbol{\phi}_{0}\right)$, respectively, 
and $[0, \omega)$ denotes the (possibly infinite) interval of existence of this solution.

Lemma 2.2. If $s_{c}$ is defined by (2.5), then for each $\boldsymbol{x}_{0}>0$ and $\boldsymbol{\Phi}_{0}<0$ the components $y(t), z(t)$ of $C\left(t ; \boldsymbol{x}_{0}, \boldsymbol{s}_{c}, \boldsymbol{\phi}_{0}\right)$ satisfy at least one of the following:

(i) $y(t)$ has minimum at some finite $t_{1}>0, y\left(t_{1}\right)=0$ and $z(t) \geqq 0$ for $0 \leqq t<\omega$;

(ii) $y(t) \downarrow 0$ and $z(t) \rightarrow \infty$ as $t \uparrow \omega$;

(iii) $z(t)$ has a minimum at some finite $t_{2}>0, z\left(t_{2}\right)=0$, and $y(t) \geqq 0$ for $0 \leqq t<\omega$;

(iv) $z(t) \downarrow 0$ and $y(t) \rightarrow \infty$ as $t \uparrow \omega$;

(v) $y(t) \downarrow \widetilde{y}$ and $z(t) \downarrow \widetilde{z}$ as $t \uparrow \infty$, where $\widetilde{y} \widetilde{z}=0$.

Proof. We consider first the case where trajectories "near" $C\left(t ; \boldsymbol{x}_{0}, s_{c}, \boldsymbol{\Phi}_{0}\right)$ exit $\bar{I}$ across the positive $z$-axis. Then by stability considerations $y\left(t ; \boldsymbol{x}_{0}, \boldsymbol{s}_{c}, \boldsymbol{\phi}_{0}\right)$ either has a zero or else approaches zero as $t \uparrow \omega$. Since $y^{\prime \prime}(t)>0$ and $z^{\prime \prime}(t)>0$ in $I$, if $y\left(t_{1} ; \boldsymbol{x}_{0}, s_{c}, \boldsymbol{\phi}_{0}\right)=0$, then it follows from (2.5) and the fact that $s_{c}\left(\boldsymbol{x}_{0}, \boldsymbol{\phi}_{0}\right) \notin\left\{\left|\boldsymbol{v}_{0}\right| \mid C\left(t ; \boldsymbol{x}_{0},\left|\boldsymbol{v}_{0}\right| \boldsymbol{\phi}_{0}\right)\right.$ exits $\bar{I}$ in finite time $\}$ that $y$ has an absolute minimum at $t_{1}$ and that (i) describes $C$. If $y\left(t ; \boldsymbol{x}_{0}, \boldsymbol{s}_{c}, \boldsymbol{\phi}_{0}\right) \downarrow 0$ and $z\left(t ; \boldsymbol{x}_{0}, s_{c}, \boldsymbol{\phi}_{0}\right) \rightarrow \infty$ as $t \uparrow \omega$, then $C$ is described by (ii). Finally, if $y\left(t ; x_{0}, s_{c}, \varphi_{0}\right) \downarrow 0$ and $z\left(t ; \boldsymbol{x}_{0}, s_{c}, \boldsymbol{\phi}_{0}\right)$ remains bounded then both $y(t)$ and $z(t)$ remain bounded in $[0, \omega)$ so that we must have $\omega=\infty$. Since it now follows from (A) that $z^{\prime \prime}>0$ in $[0, \infty)$, we must have $z\left(t ; \boldsymbol{x}_{1}, s_{c}, \boldsymbol{\varphi}_{0}\right) \downarrow \widetilde{z} \geqq 0$, which implies that $C$ satisfies (v). Analogous considerations of the cases where trajectories "near" $C\left(t ; \boldsymbol{x}_{0}, s_{c}, \boldsymbol{\phi}_{0}\right)$ exit $I$ across the positive $y$-axis lead to (iii), (iv), or (v).

Trajectories satisfying (i) or (ii) will be called $y$-critical at $t=t_{1}$ or $t=\infty$, respectively; trajectories satisfying (iii) or (iv) will be called $z$-critical at $t=t_{2}$ or $t=\infty$, respectively; finally, trajectories satisfying ( $\mathrm{v}$ ) will be called monotone. These categories are not mutually exclusive, since a trajectory can be $y$-critical at $t=t_{1}$ and then $z$-critical at $t=t_{2}>t_{1}$. Such a trajectory will be called doubly critical or, more specifically, $(y, z)$-critical at $\left(t_{1}, t_{2}\right)$; trajectories which are $(z, y)$-critical at $\left(t_{2}, t_{1}\right)$ are analogously defined when $t_{2}<t_{1}$. Our next lemma deals with the existence of such doubly critical trajectories.

LeMma 2.3. For each $\boldsymbol{x}_{0}>\mathbf{0}$ there exists a critical direction $\boldsymbol{\Phi}_{c}<0$ such that the trajectory $C\left(t ; \boldsymbol{x}_{0}, \boldsymbol{s}_{c}, \boldsymbol{\Phi}_{c}\right)$ is either doubly critical or monotone.

1 By trajectories "near" $C$ we refer to trajectories obtained by small variations in initial conditions. 
Proof. Let $\boldsymbol{x}_{0}>0$ be fixed. If $\boldsymbol{\phi}_{0}=(0,-1)$, so that $z^{\prime}(0)<0$ and $y^{\prime}(0)=0$, it follows that $y(t)>y(0)>0$ for $0<t<\omega$. Therefore for sufficiently small $\varepsilon>0$ all critical trajectories corresponding to $\boldsymbol{\phi}_{\varepsilon}=-(\varepsilon, 1-\varepsilon)$ must be $z$-critical and not $y$-critical. Analogously, for $\varepsilon$ near 1 we have $-(\varepsilon, 1-\varepsilon)$ near $(-1,0)$ and the corresponding critical trajectories must be $y$-critical and not $z$-critical. Consider now

$$
c=\sup \left\{\varepsilon \mid C\left(t ; \boldsymbol{x}_{0}, \boldsymbol{s}_{c}(\varepsilon), \boldsymbol{\phi}_{\varepsilon}\right) \text { is } z \text {-critical }\right\},
$$

where $s_{c}$ is, by virtue of its dependence on $\varphi$, also a function of $\varepsilon$. Then there will be trajectories "near" $C\left(t ; \boldsymbol{x}_{0}, \boldsymbol{s}_{c}, \boldsymbol{\Phi}_{c}\right)$ which are $z$-critical and others which are $y$-critical. It follows from stability considerations that $C\left(t ; \boldsymbol{x}_{0}, s_{c}, \boldsymbol{\phi}_{c}\right)$ itself is either doubly critical or monone.

Our final step in showing the existence of monotone trajectories requires that initial position $\boldsymbol{x}_{0}$ be expressed in terms of

$$
\left|\boldsymbol{x}_{0}\right|=|z(0)|+|y(0)| \text { and } \theta_{1}=\frac{y(0)}{\left|\boldsymbol{x}_{0}\right|}, \quad \theta_{2}=\frac{z(0)}{\left|\boldsymbol{x}_{0}\right|} .
$$

THEOREM 2.4. Given $\xi_{0}>0$, there exist $\boldsymbol{x}_{c}>\mathbf{0}, \boldsymbol{\theta}_{c}>0, s_{c}>0$, and $\boldsymbol{\Phi}_{c}<0$ such that $\left|\boldsymbol{x}_{c}\right|=\xi_{0}$ and $C\left(t ; \xi_{0}, \boldsymbol{\theta}_{c}, s_{c}, \boldsymbol{\Phi}_{c}\right)$ is a monotone trajectory.

Proof. For each $\boldsymbol{x}_{0}>\mathbf{0}$ satisfying $\left|\boldsymbol{x}_{0}\right|=\xi_{0}$ we consider a doubly critical or monotone trajectory whose existence is guaranteed by Lemma 2.3. Defining $\boldsymbol{\theta}_{\varepsilon}=(\varepsilon, 1-\varepsilon)$, we note that for sufficiently small $\varepsilon>0$ these doubly critical trajectories will be $(z, y)$-critical and approaching the initial conditions

$$
y(0)=0, z(0)=\xi_{0}, y^{\prime}(0)=0, z^{\prime}(0)=-s_{c}
$$

as $\varepsilon \rightarrow 0$. Similarly, for sufficiently small positive values of $1-\varepsilon$, the corresponding doubly critical trajectories will be $(y, z)$-critical and approaching the initial conditions

$$
y(0)=\xi_{0}, z(0)=0, y^{\prime}(0)=-s_{c}, z^{\prime}(0)=0
$$

as $\varepsilon \uparrow 1$. We therefore consider

$$
c=\sup \left\{\varepsilon \mid C\left(t ; \xi_{0}, \boldsymbol{\theta}_{\varepsilon}, s_{c}(\varepsilon), \boldsymbol{\phi}_{c}(\varepsilon) \text { is }(z, y) \text {-critical }\right\}\right. \text {. }
$$

Then $C\left(t ; \xi_{0}, \boldsymbol{\theta}_{c}, \boldsymbol{s}_{c}, \boldsymbol{\Phi}_{c}\right)$ is "near" $(y, z)$-critical trajectories and "near" other trajectories which are either $(z, y)$-critical or monotone. Since $C\left(t ; \xi_{0}, \boldsymbol{\theta}_{c}, \boldsymbol{s}_{c}, \boldsymbol{\Phi}_{c}\right)$ cannot itself be both $(y, z)$-critical and $(\boldsymbol{z}, y)$-critical, it must be monotone as asserted.

Monotone solutions as defined above satisfy either $y(t) \downarrow 0$ and 
$z(t) \downarrow \widetilde{z}$ or $y(t) \downarrow \widetilde{y}$ and $z(t) \downarrow 0$ as $t \rightarrow \infty$. The following theorem establishes conditions under which both $y(t)$ and $z(t)$ must tend to zero as $t \rightarrow \infty$.

THEOREM 2.5. If for any pair of positive constants $M$ and $m$ there exists $T>0$ such that for $t \geqq T$

$$
\begin{aligned}
& y(t) \geqq m \text { implies that } \int_{0}^{t} \int_{0}^{\tau} f_{1}(\sigma, y(\sigma), z(\sigma)) d \sigma d \tau \geqq M t \\
& z(t) \geqq m \text { implies that } \int_{0}^{t} \int_{0}^{\tau} f_{2}(\sigma, y(\sigma), z(\sigma)) d \sigma d \tau \geqq M t
\end{aligned}
$$

then every monotone trajectory for (2.1) approaches 0 as $t \rightarrow \infty$.

Proof. If $y(t) \downarrow 0$ and $z(t) \downarrow \widetilde{z}>0$ as $t \rightarrow \infty$, then

$$
z(t)>z^{\prime}(0) t+\int_{0}^{t} \int_{0}^{\tau} f_{2}(\sigma, y(\sigma), z(\sigma)) d \sigma .
$$

However, if (2.7) is satisfied we obtain the contradiction that $z(t) \rightarrow \infty$; analogously, if (2.6) is satisfied we cannot have $z(t) \downarrow 0$ and $y(t) \downarrow \widetilde{y}>0$, and this establishes the theorem.

In the special case of systems of the form (2.3) conditions (2.6) and (2.7) are implied by

$$
\int^{\infty} d(t) d t=\infty
$$

and

$$
\int^{\infty} a(t) d t=\infty \text {. }
$$

Therefore we have the following

CoROLlaRY 2.6. If (2.6)' and (2.7)' are satisfied, then every monotone solution of (2.3) satisfies $z(t) \downarrow 0$ and $y(t) \downarrow 0$ as $t \rightarrow \infty$.

3. Systems in $\boldsymbol{R}^{n}$. Our discussion of the case $n>2$ parallels that of $\S 2$ up to (2.5) where the "critical speed" $s_{c}\left(\underline{x}_{0}, \underline{\varphi}_{0}\right)$ is defined. The generalization of Lemma 2.2 is straightforward and leads to the following.

LeMma 3.1. If $s_{c}$ is defined by (2.5), then for each $\boldsymbol{x}_{0}>0$ and $\boldsymbol{\Phi}_{0}$ the components of $C\left(t ; \boldsymbol{x}_{0}, s_{c}, \boldsymbol{\phi}_{0}\right)$ satisfy at least one of the following

(i) For some $i, 1 \leqq i \leqq n, x_{i}(t)$ has a minimum for some finite $t_{i}>0, x_{i}\left(t_{i}\right)=0$, and $x_{j}(t) \geqq 0$ for $0 \leqq t<\omega$ for $j=1, \cdots, n$; 
(ii) For some $i, 1 \leqq i \leqq n, x_{i}(t) \downarrow 0$ and $x_{j}(t) \rightarrow \infty$ for some $j \neq i$ as $t \uparrow \omega$;

(iii) For all $j, 1 \leqq j \leqq n, x_{j}(t) \downarrow \widetilde{x}_{j}$ as $t \uparrow \infty$, where $\prod_{j=1}^{n} \widetilde{x}_{j}=0$.

Trajectories satisfying (i) or (ii) will be called $x_{i}$-critical at $t=t_{i}$ or $t=\infty$, respectively; trajectories satisfying (iii) will be called monotone. A trajectory which is $x_{i_{1}}$-critical at $t_{i_{1}}, x_{i_{2}}$-critical at $t_{i_{2}} \geqq t_{i_{1}}, \cdots$, and $x_{i_{k}}$-critical at $t_{i_{k}} \geqq t_{i_{k-1}}$ will be called $k$-multiplecritical or, more specifically, $\left(x_{i_{1}}, x_{i_{2}}, \cdots, x_{i_{k}}\right)$-critical.

Further progress for the case $n>2$ will require a result from combinatorial topology closely related to Sperner's lemma. Let $e_{1}, e_{2}$, $\cdots, e_{n}$ denote the vertices of an $n-1$ dimensional simplex and $\overline{e_{i_{1}} e_{i_{2}} \cdots e_{i_{n}}}$ a closed face determined by the vertices $e_{i_{1}}, \cdots, e_{i_{k}}$.

THEOREM 3.2. Let $F_{1}, \cdots, F_{n}$ denote a closed covering of $\overline{e_{1} e_{2} \cdots e_{n}}$ with the property that $F_{i}$ is disjoint from $\overline{e_{1} \cdots e_{i-1} e_{i+1} \cdots e_{n}}$ for $i=1, \cdots, n$. Then $F_{1} \cap F_{2} \cap \cdots \cap F_{n}$ is not empty.

Proof. See Alexandroff [1; p. 378].

Theorem 3.2 enables us to generalize Lemmas 2.3 and 2.4 of $\S 2$ as follows.

LEMma 3.3. For each $\boldsymbol{x}_{0}>\mathbf{0}$ there exists a critical direction $\varphi_{c}<0$ such that the trajectory $C\left(t ; \boldsymbol{x}_{0}, s_{c}, \boldsymbol{\phi}_{c}\right)$ is either n-multiplecritical or monotone.

Proof. Let $\boldsymbol{x}_{0}>0$ be fixed. If for some fixed $i, 1 \leqq i \leqq n, \varphi_{i}(0)=0$, then $x_{i}(t) \geqq x_{i}(0)>0$. Thus for $\left|\varphi_{i}(0)\right|$ sufficiently small, the critical trajectory $C\left(t ; \boldsymbol{x}_{0}, s_{c}, \boldsymbol{\Phi}_{0}\right)$ will not be $x_{i}$-critical. Now consider the $n$-1-dimensional simplex whose vertices are denoted by $e_{1}, \cdots, e_{n}$ and whose elements correspond to $\varphi_{0}$ by the relation $\boldsymbol{e}=-\boldsymbol{\phi}_{0}$. Also, for a given $x_{0}>0$, let $G_{i}$ denote the set of initial directions, expressed in terms of $\boldsymbol{e}$, for which the corresponding critical trajectory is $x_{i}$-critical or monotone. If we let $F_{i}=\bar{G}_{i}$, then $F_{i}$ is disjoint from $\overline{e_{1} \cdots e_{i-1} e_{i+1} \cdots e_{n}}$ and, by Theorem 3.2, there exists a $\boldsymbol{\Phi}_{c}\left(\boldsymbol{x}_{0}\right)$ corresponding to a vector $\boldsymbol{e}$ in $\bigcap_{i=1}^{n} F_{i}$. It follows that $C\left(t ; \boldsymbol{x}_{0}, s_{c}, \boldsymbol{\Phi}_{c}\right)$ is either monotone or else $x_{i}$-critical for all $i, 1 \leqq i \leqq n$.

In order to show the existence of a monotone trajectory we express initial position $x_{0} \geqq 0$ in terms of

$$
\left|\boldsymbol{x}_{0}\right|=\sum_{i=1}^{n}\left|x_{i}(0)\right| \text { and } \theta_{i}=\frac{x_{i}(0)}{\left|\boldsymbol{x}_{0}\right|} \text {. }
$$


TheOREM 3.4. Given $\xi_{0}>0$, there exist $\boldsymbol{x}_{c}>0, \boldsymbol{\theta}_{c}>\mathbf{0}, \boldsymbol{s}_{\mathrm{c}}>0$, and $\boldsymbol{\phi}_{c}<0$ such that $\left|\boldsymbol{x}_{c}\right|=\xi_{0}$ and $C\left(t ; \xi_{0}, \boldsymbol{\theta}_{c}, \boldsymbol{s}_{c}, \boldsymbol{\phi}_{c}\right)$ is a monotone trajectory.

Proof. Let $\left|\underline{\boldsymbol{x}}_{0}\right|$ be fixed. As $\theta_{i}(0) \downarrow 0$, the time required to become $x_{i}$-critical tends to zero. Therefore if $f_{1}, \cdots, f_{n}$ denote the vertices of the simplex whose elements correspond to $\theta_{0}$ by the relation $\theta_{0}=f$, then there exists a neighborhood of $f_{1} \overline{\cdots f_{i-1} f_{i+1} \cdots f_{n}}$ in which $n$-multiple-critical trajectories must be $x_{i}$-critical before they can become $x_{j}$-critical for all $j \neq i$. Let $G_{i}$ denote the set of $f$ for which the special solution, whose existence is guaranteed by Theorem 3.2, is either monotone or else $n$-multiple critical with the $x_{i}$ component becoming critical before any other $x_{j}$. If $F_{i}=G_{i}^{c}$, then the $F_{i}$ again provide a closed covering of ${\overline{f_{1} f_{2} \cdots f_{n}}}_{n}$ such that $F_{i}$ is disjoint from $\overline{f_{1} \cdots f_{i-1} f_{i+1} \cdots f_{n}}$ for $i=1, \cdots, n$. If $\theta_{c}$ is chosen in the (necessarily nonempty) intersection of $F_{1}, \cdots, F_{n}$, then $C\left(t ;\left|\boldsymbol{x}_{0}\right|, \boldsymbol{\theta}_{c}, \boldsymbol{s}_{c}, \boldsymbol{\Phi}_{c}\right)$ is either monotone or else simultaneously critical in $x_{1}, \cdots, x_{n}$-i.e., there exists a $t_{0}<\infty$ such that $\boldsymbol{x}\left(t_{0}\right)=\boldsymbol{x}^{\prime}\left(t_{0}\right)=0$. Since this is contrary to our assumption regarding the uniqueness of solutions, we conclude that $C\left(t ;\left|\boldsymbol{x}_{0}\right|, \boldsymbol{\theta}_{c}, s_{c}, \boldsymbol{\Phi}_{c}\right)$ is monotone.

Finally, we note that Theorem 2.5 allows a direct generalization to $n>2$.

THEOREM 3.5. If for any pair of constants $M$ and $m$ there exists $T>0$ such that for $t \geqq T$

$$
x_{i}(t) \geqq m \text { implies that } \int_{0}^{t} \int_{0}^{\tau_{i}} f_{i}(\sigma, \boldsymbol{x}(\sigma)) d \sigma d \tau \geqq M t
$$

for all $i, 1 \leqq i \leqq n$, then every monotone trajectory for (2.1) approaches 0 as $t \rightarrow \infty$.

4. Scalar differential equations. Given Theorems 2.4 and 3.4, there arises the question of the class of scalar differential equations which can be written as a second order system

$$
\boldsymbol{x}^{\prime \prime}=A(t) \boldsymbol{x}
$$

or

$$
\boldsymbol{x}^{\prime \prime}=\boldsymbol{f}(t, \boldsymbol{x}) .
$$

For $n=2$ a fairly complete answer is given in [5] where it is shown (3.1) corresponds to nonselfadjoint equations of the form

$$
\left(p_{2}(t) x^{\prime \prime}\right)^{\prime \prime}-\left(p_{1}(t) x^{\prime}\right)^{\prime}+q_{1}(t) x^{\prime}+p_{0}(t) x, \quad\left(p_{2}(t)>0\right)
$$

where the elements of $A$ are related to (3.3) by 


$$
\begin{aligned}
& a_{11}=\frac{1}{2 p_{2}}\left[p_{1}-\int_{0}^{t} q_{1}\right] ; \quad a_{12}=\frac{1}{p_{2}} \\
& a_{21}=\frac{1}{4 p_{2}}\left[p_{1}^{2}-\left(\int_{0}^{t} q_{1}\right)^{2}\right]-\frac{p_{1}^{\prime \prime}-q_{1}^{\prime}}{2}-p_{0} ; \quad a_{22}=\frac{1}{2 p_{2}}\left[p_{1}+\int_{0}^{t} q_{1}\right] .
\end{aligned}
$$

This transformation enables one to apyly Theorem 2.4 by requiring that the $a_{i j}(t)$ be continuous and appropriately positive in $[0, \infty)$. It also enables one to apply this theory to several nonlinear versions of (3.3)-e.g., the case where $p_{0}=p_{0}(t, x)$.

While there does not appear to be a general theory of such representations for the case $n>2$, it is clear that for $p_{i}>0, i=$ $0,1, \cdots, n$, the nonlinear equation

$$
\left(\left(p_{1} \cdots p_{n-1}\left(p_{n} x^{\prime \prime}\right)^{\prime \prime}\right) \cdots\right)^{\prime \prime}=p_{0}\left(t, x, x^{\prime \prime}, \cdots, x^{(2 n)}\right)
$$

can be represented in the form (3.2) by setting $x=x_{1}$ and

$$
\begin{aligned}
x_{1}^{\prime \prime} & =\frac{1}{p_{n}} x_{2} \\
x_{2}^{\prime \prime} & =\frac{1}{p_{n-1}} x_{3} \\
& \vdots \\
x_{n-1}^{\prime \prime} & =\frac{1}{p_{n}} x_{n} \\
x_{n}^{\prime \prime} & =p_{0}(t, \boldsymbol{x}) .
\end{aligned}
$$

The existence of monotone solutions of (3.4) corresponds to solutions of (3.4) which are strictly decreasing together with their second, fourth, $\cdots, 2 n$th derivatives, while $x^{\prime}, x^{\prime \prime \prime}, \cdots, x^{(2 n-1)}$ are increasing. Theorem 3.5 establishes further criteria which assure that $x, x^{\prime \prime}, \cdots$, $x^{(2 n)}$ all tend to zero.

Added in proof. A different technique for generalizing the results of [3] is contained in Gunnar Aronsson's "On two theorems by Hartman and Winter. An application of the Wazewski retract method" soon to appear in J. Math. Anal. and Appl.

\section{REFERENCES}

1. P. Alexandroff, Topologie, Springer (Berlin), 1935.

2. P. Hartman, Ordinary Differential Equations, Wiley (New York), 1964.

3. P. Hartman and A. Wintner, On monotone solutions of systems of nonlinear differential equations, Amer. J. Math., 77 (1955), 860-866.

4. K. Kreith, Oscillation Theory, Springer Lecture Notes \#324 (Berlin, Heidelberg), 1973.

5. — A nonselfadjoint dynamical system, Proc. Edinburgh Math. Soc., 19 (1974), 77-87. 
6. W. T. Reid, Principal solutions of nonoscillatory linear differential systems, J. Math. Anal. and Applications, 9 (1964), 397-423.

Received June 10, 1977 and in revised form May 3, 1978.

UNIVERSITY OF CALIFORNIA

DAvis, CA 95616 



\section{PACIFIC JOURNAL OF MATHEMATICS}

EDITORS

DONALD BABBITT (Managing Editor)

University of California

Los Angeles, California 90024

HUGo RossI

University of Utah

Salt Lake City, UT 84112

C. C. MOORE

J. DUGUNDJI

Department of Mathematics

University of Southern California

Los Angeles, California 90007

R. FinN AND J. MiLgraM

Stanford University

Stanford, California 94305

University of California

Berkeley, CA 94720

\section{ASSOCIATE EDITORS}

E. F. BrCKENBACH

B. H. NeUmanN

F. WOLF

K. YoshidA

\section{SUPPORTING INSTITUTIONS}

UNIVERSITY OF BRITISH COLUMBIA

UNIVERSITY OF SOUTHERN CALIFORNIA

CALIFORNIA INSTITUTE OF TECHNOLOGY

STANFORD UNIVERSITY

UNIVERSITY OF CALIFORNIA

UNIVERSITY OF HAWAII

MONTANA STATE UNIVERSITY

UNIVERSITY OF TOKYO

UNIVERSITY OF NEVADA, RENO

UNIVERSITY OF UTAH

NEW MEXICO STATE UNIVERSITY

WASHINGTON STATE UNIVERSITY

OREGON STATE UNIVERSITY

UNIVERSITY OF WASHINGTON

UNIVERSITY OF OREGON 


\section{Pacific Journal of Mathematics}

\section{Vol. 81, No. $1 \quad$ November, 1979}

Thomas E. Armstrong, Simplicial subdivision of infinite-dimensional compact cubes ..................................... 1

Herbert Stanley Bear, Jr., Approximate identities and pointwise convergence ................................

Richard David Bourgin, Partial orderings for integral representations on convex sets with the Radon-Nikodým property..................

Alan Day, Herbert S. Gaskill and Werner Poguntke, Distributive lattices

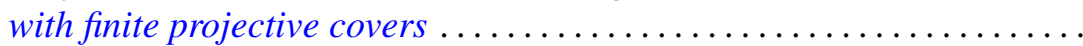

Heneri Amos Murima Dzinotyiweyi and Gerard L. G. Sleijpen, A note on measures on foundation semigroups with weakly compact orbits ......

Ronald James Evans, Resolution of sign ambiguities in Jacobi and Jacobsthal sums ...................................

John Albert Fridy, Tauberian theorems via block dominated matrices ......

Matthew Gould and Helen H. James, Automorphism groups retracting onto

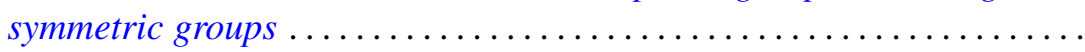

Kurt Kreith, Nonlinear differential equations with monotone solutions . . . . 101

Brian William McEnnis, Shifts on indefinite inner product spaces........ 113

Joseph B. Miles, On entire functions of infinite order with radially

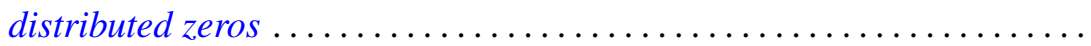

Janet E. Mills, The idempotents of a class of 0-simple inverse semigroups ...............................

Edward Jean Moulis, Jr., Generalizations of the Robertson functions ...

Richard A. Moynihan and Berthold Schweizer, Betweenness relations in probabilistic metric spaces.......................

Stanley Ocken, Perturbing embeddings in codimension two ....

Masilamani Sambandham, On the average number of real zeros of a class of random algebraic curves.

Jerry Searcy and B. Andreas Troesch, A cyclic inequality and a related eigenvalue problem.

Roger R. Smith and Joseph Dinneen Ward, $M$-ideals in $B\left(l_{p}\right)$...

Michel Talagrand, Deux généralisations d'un théorème de I. Namioka ..

Jürgen Voigt, $O n Y$-closed subspaces of $X$, for Banach spaces $X \subset Y$;

existence of alternating elements in subspaces of $C(J)$

Sidney Martin Webster, On mapping an $n$-ball into an $(n+1)$-ball in complex spaces

David J. Winter, Triangulable subalgebras of Lie p-algebras ... 